\title{
Proceeding
}

Supplementary Issue: Winter Conferences of Sports Science. Costa Blanca Sports Science Events, 24 April 2020. Alicante, Spain.

\section{An analytical study of some biomechanical variables on the short, medium and long-distance treadmill for Iraqi heroes}

\author{
HAIDER FAYYADH ALAMIRI ${ }^{4}$, GHAFAR SAEED ISSA ${ }^{2}$, MAJED HASSAN² \\ ${ }^{1}$ Faculty of Physical Education and Sport Sciences, University of Kufa, Iraq \\ ${ }^{2}$ Faculty of Physical Education and Sport Sciences, University of Wasit, Iraq
}

\begin{abstract}
Kinetic analysis is considered an important science, which mainly depends on the use of laws and foundations used in biomechanics for the purpose of studying movement anatomically and mechanically. This research provides an overview of the various tasks involved in analysing movement of the human body. which relates to the analysis of the movement of athletes and their tracking to understand the most general behaviours of the athlete during jogging, it is noted that there are many mechanical variables that play a great role in achieving achievement for the athlete during the race including the length, frequency and time of the step as well as the maximum force of the exerts on the field the amount of pressure applied to the foot by the athlete during the racing stages. The researcher used the descriptive analytical method to process data and information related to the nature of the problem and the researcher chose a sample consisting of six Iraqi champions in a competition $(100 \mathrm{~m}, 1500 \mathrm{~m}, 5000 \mathrm{~m})$ in a deliberate way, two for each competition. For the time period from $9 / 11 / 2018$ to $1 / 12 / 2018$ in the biomechanical laboratory in the University of Kufa, using a scanner (gait analysis) and a time and length variable was chosen because it has A major role in the sports movement and its effectiveness during the race. the researcher concluded that the running rhythm for time and step length will be distributed in a balanced and according to the requirements of the race.

Keywords: Motion analysis; Biomechanical; Treadmill; Athletic performance.

\section{Cite this article as:}

Alamiri, H.F., Issa, G.S., \& Hassan, M. (2020). An analytical study of some biomechanical variables on the short, medium and long-distance treadmill for Iraqi heroes. Journal of Human Sport and Exercise, 15(2proc), S139S145. doi:https://doi.org/10.14198/ihse.2020.15.Proc2.03

Corresponding author. Faculty of Physical Education and Sport Sciences, University of Wasit, Iraq.

E-mail: dr.ghafarsaeedissa@gmail.com

Supplementary Issue: Winter Conferences of Sports Science. Costa Blanca Sports Science Events, 24 April 2020. Alicante, Spain.

JOURNAL OF HUMAN SPORT \& EXERCISE ISSN 1988-5202

(c) Faculty of Education. University of Alicante

doi:10.14198/jhse.2020.15.Proc2.03
\end{abstract}




\section{INTRODUCTION}

The dynamic analysis is an important science, which depends mainly on the use of laws and foundations used in biochemical science for the purpose of studying movement anatomically and mechanically, which is essentially an entry point to know the behaviour of human movement or its course, and that the great development that has taken place in the manufacture of devices such as the cameras which helped to identify many important variables in the sports field'.

This research provides an overview of the various tasks involved in the movement analysis of the human body, which relates to analysing the movement of athletes and tracking them to understand the most general behaviours of athletes while running ${ }^{2}$. This research provides a comprehensive survey on the analysis of mathematical movement based on the use of computers (Hughes and Bartlett, 2015) and that the platform (Zebris FDM) recognized at the national and international levels as a reference guide in identifying many of the variables of arena games ${ }^{3}$.

This research demonstrates how these techniques are used to study biomechanical variables by first looking at the underlying factors involved in mechanical jogging performance and thus showing current differences between runners while running for short, medium and long distances 4 .

It is noticeable that there are many mechanical variables that play a big role in achieving the athletic accomplishment during the race, including the length of the step, its frequency and the time of the step, as well as the maximum amount of force exerted during the run and the amount of pressure exerted by the athlete and the corners of the joints of the body parts during the stages of the race.

These biomechanical factors play a role in explaining the difference between individuals as well as the economy of on-the-go effort 5 . The purpose of this study is to identify the current state of the runners and the relationship between the time variable study and the length of the step for short, medium and long distance runners, and this study is not intended to provide a comprehensive analysis of all anatomical and mechanical factors in jogging ${ }^{6}$. The importance of studying the time variable and step length in the competitions (100 m, $1500 \mathrm{~m}$ and $5000 \mathrm{~m}$ ) is that it is more effective among other variables in achieving accomplishment during the race with different races from each race according to the requirements of the event ${ }^{7}$. So, the researcher studied the time variable and length of the step for its great importance among the runners in the races of $(100 \mathrm{~m}, 1500$ and $5000 \mathrm{~m})$ among the Iraqi heroes. The researchers designed this study in a scientific procedure using $\mathrm{h} / \mathrm{p} / \mathrm{cosmos}$ device from the German company (Zebris) by reading the data in a standardized

\footnotetext{
${ }^{1}$ McGarry, T. (2009) .Applied and theoretical perspectives of performance analysis in sport: Scientific issues and challenges: International Journal of Performance Analysis in Sport. Taylor \& Francis, 9(1), pp. 128-140.

2 Robinson, G. \& O'Donoghue, P. (2007) .A weighted kappa statistic for reliability testing in performance analysis of sport: International Journal of Performance Analysis in Sport. Taylor \& Francis, 7(1), pp. 12-19.

${ }^{3}$ Hughes, M. \& Franks, I. M. (2004) .Notational analysis of sport: Systems for better coaching and performance in sport. Psychology Press.

${ }^{4}$ Bartlett, R. (2007). Introduction to Sports Biomechanics, Analysing Human Movement Patterns. Routledge.

${ }^{5}$ More, K. E. N. \& Franks, I. A. N. M. (2004) .Measuring coaching effectiveness :in Notational analysis of Sport. Routledge, pp. 259-272.

${ }^{6}$ Podlubny, I. et al. (2013) .Matrix approach to discrete fractional calculus III: non-equidistant grids, variable step length and distributed orders, Philosophical Transactions of the Royal Society A: Mathematical, Physical and Engineering Sciences. The Royal Society Publishing, 371(1990), p. 20120153.

7 Butterworth, A. \& O' Donoghue, P. and Cropley, B. (2013). Performance profiling in sports coaching: a review: International Journal of Performance Analysis in Sport. Taylor \& Francis, 13(3), pp. 572-593.
} 
way during testing ${ }^{8}$. This study aimed to determine the variable values of the races $(100 \mathrm{~m}, 1500 \mathrm{~m}$ and 5000 $\mathrm{m})$.

\section{MATERIAL AND METHODS}

\section{Procedures}

The researcher used the analytical descriptive method to process data and information about the nature of the problem. The causal method is in which the researcher tries to determine the cause of differences in the behaviour or condition of a group of individuals, which means that the observed groups differ among themselves in attributing certain variables 9 . The purpose of regulating this information and classification is to help the researcher to reach conclusions and generalizations that help us develop the reality that we study, and the descriptive way in which to reach conclusions that contribute to understanding this reality and developing it ${ }^{10}$.

\section{Participants}

The researcher chose to six Iraqi heroes sample in the competitions of $(100 \mathrm{~m}, 1500 \mathrm{~m}, 5000 \mathrm{~m})$ two for each competition in a deliberate way. For the time period from September 11, 2018 to 1/12/2018. In the Biomechanics Laboratory in the Faculty of Physical Education and Sports Sciences / Kufa University, the variable time and length of the step was chosen because it has a big role in the sports movement and its effectiveness during the race ${ }^{11}$, thus based on the experience of the researcher who was a former athlete in arena games competitions as well as professor of motor analysis in the Faculty of Physical Education and Sports Sciences at Kufa University, The test was conducted at the Biomechanics laboratory on 11 of September 2018 at 4:00 pm in the presence of the auxiliary team and the runners of $(100 \mathrm{~m}, 1500 \mathrm{~m}, 5000$ $\mathrm{m})$ races. The speed at which the test was conducted was determined on the time variable and the length of the step that the runner could take during the race ${ }^{12}$. The speed limit was $10 \mathrm{~km} / \mathrm{h}$ on the machine for the race $(5000 \mathrm{~m})$, the speed of $16 \mathrm{~km} / \mathrm{h}$ for the race $(1500 \mathrm{~m})$ and the speed of $22 \mathrm{~km} / \mathrm{h}$ for the race distance $(100 \mathrm{~m})$. These speeds were chosen according to the times achieved by the contestants in the official competitions, by dividing the total distance by time to extract the speed rate $(\mathrm{m} / \mathrm{s})$ and then multiplying it by (3.6) to convert to $\mathrm{km}$, because the speed on the walking platform of the device that deals with the units of the kilometre, the analyser machine has different speeds starting (from $1 \mathrm{~km}$ to $22 \mathrm{~km}$ ) per hour, the device works when the runner reaches the required speed for each running test.

\section{Measures}

The data is recorded by the cable contact with the platform to the calculator that includes the application of the platform (Zebris FDM). It then takes 10 seconds to register on the device to get the data during the test.

\section{Analysis}

The researcher use the appropriate statistic finder to solve the research problem and get the results using the statistical analysis program (SPSS version 20).

\footnotetext{
8 Brunner, W. (2014) . Gait analysis system. Google Patents.

9 Harriss, D. J,\& MacSween, A. \& Atkinson, G. (2017) .Standards for ethics in sport and exercise science research: 2018 update: International journal of sports medicine. ( ) Georg Thieme Verlag KG, 38(14), pp. 1126-1131.

${ }^{10}$ Blessing, L. T. M. and Chakrabarti, A. (2009) DRM: A design reseach methodology. Springer.

11 Havlík, J. and Straka, O. (2015) .Performance evaluation of iterated extended Kalman filter with variable step-length: in Journal of Physics: Conference Series. IOP Publishing, p. 12022.

12 Kahveci, T. \& Singh, A. (2001) .Variable length queries for time series data: in Proceedings 17th International Conference on Data Engineering. IEEE, pp. 273-282.
} 


\section{RESULTS}

Table 1. Shows the arithmetic average and the standard deviation of the time variable and the step length of the foot (left and right).

\begin{tabular}{lccccccc}
\hline \multirow{2}{*}{ Variable name } & Right & \multicolumn{2}{c}{ Speed $\mathbf{1 0}$ km/h } & \multicolumn{2}{c}{ Speed 16 kmlh } & \multicolumn{2}{c}{ Speed 22 km/h } \\
\cline { 2 - 8 } & Left & Mean & SD & Mean & SD & Mean & SD \\
\hline \multirow{2}{*}{ Step length (cm) } & L & 97.6 & 6.80 & 135 & 0.70 & 148 & 0.70 \\
& $\mathrm{R}$ & 96.6 & 7.09 & 139.5 & 1.41 & 143 & 1.41 \\
Step time (sec) & $\mathrm{L}$ & 0.35 & 0.07 & 0.30 & 0.00 & 0.20 & 0.00 \\
& $\mathrm{R}$ & 0.35 & 0.07 & 0.30 & 0.00 & 0.20 & 0.00 \\
\hline
\end{tabular}

\section{Gait parameters}

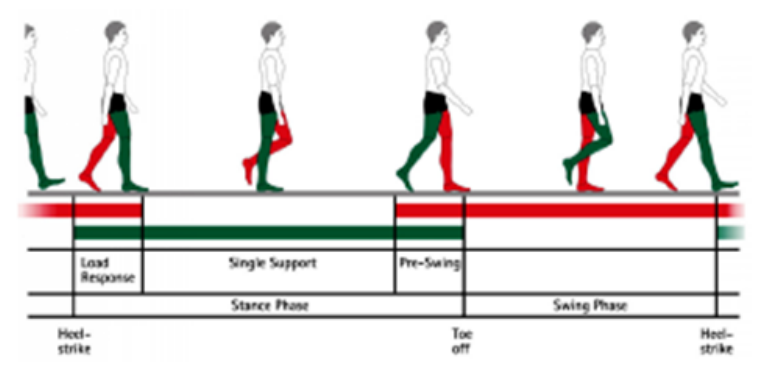

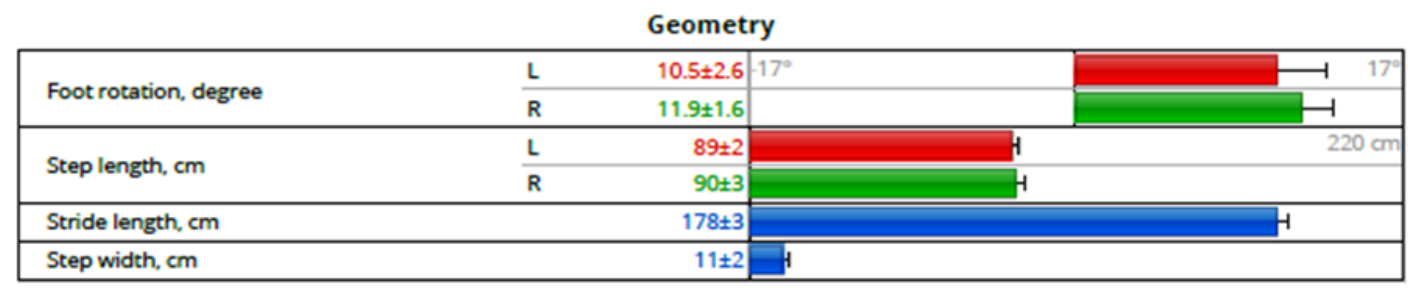

\begin{tabular}{|c|c|c|c|c|}
\hline \multicolumn{5}{|c|}{ Phases } \\
\hline \multirow{2}{*}{ Stance phase, \% } & $L$ & $36.3 \pm 1.4$ & H & $100 \%$ \\
\hline & $R$ & $36.7 \pm 2.3$ & $\mathrm{H}$ & \\
\hline \multirow{2}{*}{ Load response, \% } & $L$ & $0.0 \pm 0.0$ & & \\
\hline & $\mathbf{R}$ & $0.0 \pm 0.0$ & & \\
\hline \multirow{2}{*}{ Mid stance, \% } & $L$ & $36.3 \pm 1.4$ & 4 & \\
\hline & $\mathbf{R}$ & $36.5 \pm 2.1$ & -1 & \\
\hline \multirow{2}{*}{ Pre-Swing \% } & $L$ & $0.0 \pm 0.0$ & & \\
\hline & $\mathbf{R}$ & $0.0 \pm 0.0$ & & \\
\hline \multirow{2}{*}{ Swing phase, \% } & $L$ & $63.7 \pm 1.4$ & \multicolumn{2}{|c|}{$\mathrm{H}$} \\
\hline & $\mathbf{R}$ & $63.3 \pm 2.3$ & \multicolumn{2}{|c|}{ H } \\
\hline \multirow[t]{2}{*}{ Double stance phase, \% } & & $0.0 \pm 0.0$ & & \\
\hline & \multicolumn{4}{|c|}{ TImIng } \\
\hline \multirow{2}{*}{ Step time, sec } & $L$ & $0.3 \pm 0.0$ & \multicolumn{2}{|r|}{$0.8 \mathrm{sec}$} \\
\hline & $\mathbf{R}$ & $0.3 \pm 0.0$ & \multicolumn{2}{|l|}{ H } \\
\hline
\end{tabular}

Figure 1. Measurement time variable and length of the step.

Table 1 shows the average arithmetic and standard deviation of biomechanical variables, the time variable and the runner footstep length (right-left) of $(100 \mathrm{~m}, 1500 \mathrm{~m}$, and $5000 \mathrm{~m}$ ) races for six runners, during each competition. The researcher found that there is a difference in the time variable and step length for each runner depending on the kind of race they take and the limb whether it was right or left as shown in Figure 1. 
The researcher attributed the reason for this difference to the nature of the competitions in terms of distance and the runners running mechanism as well as the requirements of the race that vary from race to race in terms of speed and time.

\section{DISCUSSION}

When studying the speed law, which represents the ratio between the distance travelled by the body to the time of the distance, it is possible to identify many physical and training features that we can develop by training the player in accordance with the speed law ${ }^{13}$. Sports achievement is influenced by several mechanical variables, namely the average speed, which is related to both the distance and time of the race ${ }^{14}$. On the other hand, the achievement is related to the characteristics and components of the step, which are related to the physical characteristics related to the application of mechanical conditions ${ }^{15}$. The speed rate is the product of both step length and repetition, since the speed of the runner is his ability to perform successive movements of one type with specific distances ${ }^{16}$. The step length variable is the total distance of the race divided by the number of steps achieved in that distance, as for the frequency of the step it is the result of the divided number of steps by the total time of that distance. The researcher noted that the length of the step is important in runners at a short distance because the great power exercised by the runners to get a big push during the race ${ }^{17}$, is also a rapid frequency step and because of rapid nerve impulses sent by the nervous system to increase the rhythm of the steps and thus will lead to an increase in the speed rate because its time is too short to achieve a large force thrust and according to the momentum of the mechanical equation $=$ force $x$ time ${ }^{18}$. Through analysis of the data by the researcher it was found that the variable step length for mid-distance runners is less than the length of the step for the short distance runners, because the amount of force exerted by runners is low compared to the strength exerted by short-distance runners enough to match the nature of the race ${ }^{19}$. The reason for this is that the instructions sent by the nervous system to the lower limbs are compatible with the conditions of the race, as well as to distribute the effort exerted by the athlete during the race and prevent fatigue. So, the average velocity depends on the step length and time variable ${ }^{20}$. As for the long-distance runners, the length of the step is less than the length of the short and medium-distance runners step. The reason is that the force exerted is low by the long distance runner, thus the power produced by the runners is commensurate with the nature of the race, which needs to be heavily shouldered with the most among of effort saving and the distribution of the float along the race, and energy distribution along the race ${ }^{21}$. Another reason is that the signals sent by the nervous system to the lower limbs

\footnotetext{
${ }^{13}$ Cabooter, D. et al. (2011) .Fast method development of rooibos tea phenolics using a variable column length strategy: Journal of Chromatography A. Elsevier, 1218(41), pp. 7347-7357.

14 Havlík, J. and Straka, O. (2015).Performance evaluation of iterated extended Kalman filter with variable step-length: in Journal of Physics: Conference Series. IOP Publishing, p. 12022.

15 Calvo, M. P. \& Sanz-Serna, J. M. (1993).The development of variable-step symplectic integrators, with application to the twobody problem: SIAM Journal on Scientific Computing. SIAM, 14(4), pp. 936-952.

${ }^{16} \mathrm{Hino}, \mathrm{T}$. \& Maeno, T. (2004) .Development of a miniature robot finger with a variable stiffness mechanism using shape memory alloy: International Symposium on Robotics and Automation, Querétaro, México, Aug, pp. 25-27.

${ }_{17}^{17}$ Pontzer, H. (2005). A new model predicting locomotor cost from limb length via force production: Journal of Experimental Biology. The Company of Biologists Ltd, 208(8), pp. 1513-1524.

${ }^{18}$ Hall, S. (2014) .Basic biomechanics: McGraw-Hill Higher Education.

${ }^{19}$ Herzog, W. and ter Keurs, H. E. D. J. (1988) .Force-length relation of in-vivo human rectus femoris muscles: Pflügers Archiv. Springer, 411(6), pp. 642-647.

20 Tartaruga, M. P. et al. (2012). The relationship between running economy and biomechanical variables in distance runners: Research Quarterly for Exercise and Sport. Taylor \& Francis, 83(3), pp. 367-375.

${ }^{21}$ Slawinski, J. et al. (2001) .Effect of supra-lactate threshold training on the relationship between mechanical stride descriptors and aerobic energy cost in trained runners: Archives of physiology and biochemistry. Taylor \& Francis, 109(2), pp. 110-116.
} 
are suitable for racing conditions and this requires the distribution of effort during the race for all the body systems, so the speed rate is low during the race 22 .

\section{CONCLUSION}

The variable time and length of the step produced by the racers varies from short, medium and long distances runners due to the different distance of the race, as well as the rhythm of the run for the time and length of the step length variable which is distributed in a balanced way according to the race requirement and the time and step length depends on the race speed where is as the race was faster the step length is longer and the step time is shot because the speed has a reverse relationship with time thus the time and step length depends also on the length of runner leg which is related to the which is related to the genetic factor of the athlete who plays a major role in accomplishing the race.

\section{REFERENCES}

Bartlett, R. (2007). Introduction to Sports Biomechanics, Analysing Human Movement Patterns. Routledge.

Blessing, L. T. M. and Chakrabarti, A. (2009). DRM: A design reseach methodology. Springer.

Brunner, W. (2014). Gait analysis system. Google Patents.

Butterworth, A. \& O' Donoghue, P. and Cropley, B. (2013). Performance profiling in sports coaching: a review: International Journal of Performance Analysis in Sport. Taylor \& Francis, 13(3), pp. 572-593. https://doi.org/10.1080/24748668.2013.11868672

Cabooter, D. et al. (2011). Fast method development of rooibos tea phenolics using a variable column length strategy: Journal of Chromatography A. Elsevier, 1218(41), pp. 7347-7357. https://doi.org/10.1016/j.chroma.2011.08.069

Calvo, M. P. \& Sanz-Serna, J. M. (1993). The development of variable-step symplectic integrators, with application to the two-body problem: SIAM Journal on Scientific Computing. SIAM, 14(4), pp. 936952. https://doi.org/10.1137/0914057

Hall, S. (2014). Basic biomechanics: McGraw-Hill Higher Education.

Harriss, D. J,\& MacSween, A. \& Atkinson, G. (2017). Standards for ethics in sport and exercise science research: 2018 update: International journal of sports medicine. (C) Georg Thieme Verlag KG, 38(14), pp. 1126-1131. https://doi.org/10.1055/s-0043-124001

Havlík, J. and Straka, O. (2015). Performance evaluation of iterated extended Kalman filter with variable step-length: in Journal of Physics: Conference Series. IOP Publishing, p. 12022. https://doi.org/10.1088/1742-6596/659/1/012022

Herzog, W. and ter Keurs, H. E. D. J. (1988). Force-length relation of in-vivo human rectus femoris muscles: Pflügers Archiv. Springer, 411(6), pp. 642-647. https://doi.org/10.1007/bf00580860

Hino, T. \& Maeno, T. (2004). Development of a miniature robot finger with a variable stiffness mechanism using shape memory alloy: International Symposium on Robotics and Automation, Querétaro, México, Aug, pp. 25-27.

Hughes, M. \& Bartlett, R. (2015). What is performance analysis?: in Essentials of performance analysis in sport. Routledge, pp. 18-28.

Hughes, M. \& Franks, I. M. (2004). Notational analysis of sport: Systems for better coaching and performance in sport. Psychology Press. https://doi.org/10.4324/9780203641958

$22 \mathrm{Wu}, \mathrm{L} . \mathrm{M}$. N. et al. (2012). Increasing internodal distance in myelinated nerves accelerates nerve conduction to a flat maximum: Current Biology. Elsevier, 22(20), pp. 1957-1961. 
Kahveci, T. \& Singh, A. (2001). Variable length queries for time series data: in Proceedings 17th International Conference on Data Engineering. IEEE, pp. 273-282. https://doi.org/10.1109/icde.2001.914838

McGarry, T. (2009). Applied and theoretical perspectives of performance analysis in sport: Scientific issues and challenges: International Journal of Performance Analysis in Sport. Taylor \& Francis, 9(1), pp. 128-140. https://doi.org/10.1080/24748668.2009.11868469

More, K. E. N. \& Franks, I. A. N. M. (2004).Measuring coaching effectiveness :in Notational analysis of Sport. Routledge, pp. 259-272.

Podlubny, I. et al. (2013). Matrix approach to discrete fractional calculus III: non-equidistant grids, variable step length and distributed orders, Philosophical Transactions of the Royal Society A: Mathematical, Physical and Engineering Sciences. The Royal Society Publishing, 371(1990), p. 20120153. https://doi.org/10.1098/rsta.2012.0153

Pontzer, H. (2005). A new model predicting locomotor cost from limb length via force production: Journal of Experimental Biology. The Company of Biologists Ltd, 208(8), pp. 1513-1524. https://doi.org/10.1242/jeb.01549

Robinson, G. \& O'Donoghue, P. (2007). A weighted kappa statistic for reliability testing in performance analysis of sport: International Journal of Performance Analysis in Sport. Taylor \& Francis, 7(1), pp. 12-19. https://doi.org/10.1080/24748668.2007.11868383

Slawinski, J. et al. (2001). Effect of supra-lactate threshold training on the relationship between mechanical stride descriptors and aerobic energy cost in trained runners: Archives of physiology and biochemistry. Taylor \& Francis, 109(2), pp. 110-116. https://doi.org/10.1076/apab.109.2.110.4270

Tartaruga, M. P. et al. (2012). The relationship between running economy and biomechanical variables in distance runners: Research Quarterly for Exercise and Sport. Taylor \& Francis, 83(3), pp. 367375. https://doi.org/10.1080/02701367.2012.10599870

Wu, L. M. N. et al. (2012). Increasing internodal distance in myelinated nerves accelerates nerve conduction to a flat maximum: Current Biology. Elsevier, 22(20), pp. 1957-1961. https://doi.org/10.1016/j.cub.2012.08.025 